\title{
Future, Opportunities and Challenges of Inkjet Technologies
}

\author{
J.R. Castrejón-Pita ${ }^{1}$, W.R.S. Baxter ${ }^{2}$, J. Morgan ${ }^{3}$, S. Temple ${ }^{4}$, G.D. Martin ${ }^{1}$, and I.M. Hutchings ${ }^{1}$ \\ ${ }^{1}$ Inkjet Research Centre, University of Cambridge, \\ 17 Charles Babbage Road, Cambridge, CB3 OFS, United Kingdom \\ ${ }^{2}$ Inca Digital Printers Ltd, 515 Coldhams Lane, Cambridge, CB1 3JS, United Kingdom \\ ${ }^{3}$ Domino Printing Sciences Plc, Bar Hill, Cambridge, CB23 8TU, United Kingdom and \\ ${ }^{4}$ Templetech Ltd, 10 Cambridge Road, Impington, CB24 9NU, United Kingdom
}

\begin{abstract}
Inkjet printing relies on the formation of small liquid droplets to deliver precise amounts of material to a substrate under digital control. Inkjet technology is becoming relatively mature and is of great industrial interest thanks to its flexibility for graphical printing and its potential use in less conventional applications such as additive manufacturing and the production of printed electronics and other functional devices. Its advantages over traditional methods of printing include the following: it produces little or no waste, it is versatile because several different methods exist, it is non-contact and does not require a master template so that printed patterns can be readily modified on demand. However, the technology is in need of further development to become mainstream in emerging applications such as additive manufacturing (3D printing). This review contains a description of conventional and less common inkjet methods and surveys the current applications of inkjet in industry. This is followed by specific examples of the barriers, limitations and challenges faced by inkjet in both graphical printing and manufacturing.
\end{abstract}

Accepted for publication in Atomization and Sprays (2013).

Available at: https://www.repository.cam.ac.uk/handle/1810/238990

Keywords: inkjet, drop on demand, continuous jet, droplet.

\section{INTRODUCTION}

Inkjet printing can be considered an umbrella term covering a range of technologies all of which involve the ejection of droplets of ink from a printhead on to a substrate. Since the 1970 s when inkjet printing was first commercialised, it has provided a method for delivering and positioning precise small volumes of liquid at high repetition rates under digital control. A key feature of all embodiments of this printing method is that it is noncontact. Whilst inkjet technology has great value for printing purposes, it also has the potential to act as an element of manufacturing processes, whether in the form of accurate dispensing, as can be used for pharmaceutical, bio-chemical or chemical applications, or for building three-dimensional objects from engineering materials (so-called 3D printing, an example of additive manufacturing) so long as they can be supplied in liquid form. The future of inkjet in its traditional niche of printing is still very promising, as the market for graphical printing is very valuable. In principle, inkjet can replace a variety of conventional methods of printing in a large number of commercial applications. Regardless of these extensive potential uses, however, the adoption of inkjet has so far been concentrated in just a few such applications. Several inkjet methods exist that operate under very different principles and utilize diverse inks for various applications. This variety of methods in part reflects the fact that inkjet printing has some fundamental limitations and that these limitations have a different impact on each of the various methods employed to eject droplets. The barriers to wider adoption of inkjet in non-traditional applications involve both technical and commercial issues. A brief survey of current inkjet processes is presented below and followed by some specific examples of obstacles to the adoption of inkjet.

\section{EXAMPLES OF INKJET TECHNOLOGIES}

Many different techniques fall under the generic title "inkjet". The most basic common feature is that some kind of liquid (possibly also containing particles) is transported from a printhead, across a gap, to a receiving substrate. The liquid then, either as it is, or after subsequent modification (e.g. by drying, curing, absorption etc.), performs some function (e.g. forms an image on paper or a conductive structure on an insulating substrate). Another feature, shared by all inkjet systems, is an ability to control the flow of the liquid in a precise way so that a known small volume of material is deposited on to a predetermined location. This control is often achieved by choosing to release a drop, or a stream of drops, from a printhead in which the diameter of the drop is usually related to the size of the nozzle. There are inkjet systems in which the volume dispensed is better described as a slug, a jet or a spray of liquid. In general, two principal attributes make inkjet systems attractive: the non-contact deposition of material and the precise control of the amount and placement of that material. Exactly what is or is not regarded as an inkjet system is sometimes a matter of context. For example a hand-held air brush would not be considered to be an inkjet system, but a similar computer-controlled airbrush used to create a graphical image on a rotating drum might well be 
considered an inkjet system by some. Detailed descriptions of inkjet technologies can be found in chapter II in [1], but brief descriptions of some of these are given below.

\section{A. Conventional inkjet technologies}

There are two main groups of conventional inkjet technologies: drop on demand (DoD) and continuous inkjet (CIJ), [2] and [3]. Both techniques, by different methods, produce uniformly-sized droplets and their most common application is in graphical printing. In drop on demand mode, liquid droplets are produced in response to a digital signal or waveform. In typical industrial applications, this technique is used to produce $15-55 \mu \mathrm{m}$ diameter droplets, with drop velocities ranging from 3 to $15 \mathrm{~m} / \mathrm{s}$ and printing frequencies of up to $100 \mathrm{kHz}$. In contrast, in CIJ, a continuous stream of liquid is stimulated to break up into drops by the action of a harmonic modulation. In this mode, the separation of the drops is determined by the modulation frequency and the speed of the jet. Continuous inkjet systems typically produce $80-100 \mu \mathrm{m}$ droplets travelling at speeds of 20 $\mathrm{m} / \mathrm{s}$ with drop frequencies which can exceed $250 \mathrm{kHz}$. In the most common implementation of CIJ printing, electrostatic charging and deflection are used to select and steer individual drops to define the final printed pattern. For both DoD and CIJ, several actuation methods exist; piezolectric, thermal and electromagnetic actuators are the most common.

Apart from these common printing modes, other methods can also be used to produce droplets. Most of these have been developed in response to a particular industrial need, such as for the printing of very small droplets, or for colloidal inks. Brief descriptions of some of these technologies are given below.

\section{B. Other inkjet methods}

This section considers inkjet processes not based on the more conventional DOD or CIJ techniques described above. The conventional processes continue to be developed but a quite different approach may suit some applications better: for example, the generation of very small deposit volumes, the deposition of liquid containing a high loading of particles, very high speed printing or the deposition of delicate biological materials.

\section{Kodak Stream}

This technology is an array-based, continuous inkjet process which involves some significant differences from conventional CIJ systems, which may have advantages for certain applications, $[4,5]$. Rather than using an acoustic means to disturb the jets and cause break-up

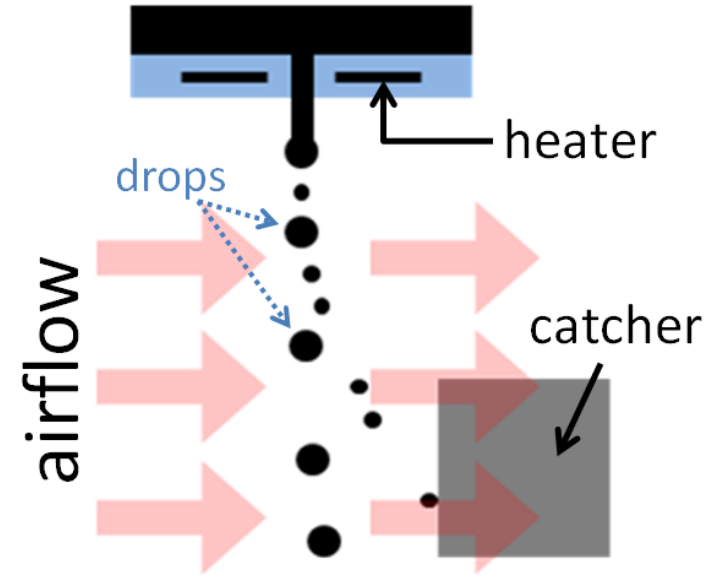

FIG. 1: (Colour online) Operation of Stream inkjet process.

into drops, each nozzle has a small heating element associated with it which can be modulated to change the temperature of the liquid jet at the nozzle, and hence its local surface tension. This introduces surface tension gradients with Marangoni forces driving liquid flow away from the hot regions and hence controlling the break-up of the jet. In addition, drops are selected to print by adjusting the timing of the heat pulse such that either small drops or large drops are created. As the drop streams move towards the substrate the small drops are removed by a transverse air flow leaving the large drops to be deposited on to the substrate. This is illustrated in Fig. 1. The nozzle and heater system is fabricated using siliconbased MEMS technology. Although the system described here seems to be that embodied in the current Kodak product, other publications from the company describe alternatives such as the use of an asymmetric heater at the nozzle to direct the stream [6], perhaps acting as an alternative to the air flow for selecting drops.

Being a continuous process, this system can operate at drop delivery rates which are significantly higher than those from DOD systems, thus potentially enabling printing at very high speeds.

\section{Silverbrook}

Although the current version of the Silverbrook technology employs a variation of the common thermal (vapour bubble) actuation it has a number of features which make it stand out from other examples, [7]. Not least among these is the use of silicon-based microelectromechanical (MEMs) fabrication techniques to construct the whole printhead in the form of a stackable chip which enables high nozzle densities and wide nozzle arrays. The print format is usually an array placed across the width of a continuously moving substrate. The examples demonstrated by the company (some of which are commercially available) include label printers, small home/office printers, poster printers and high volume 


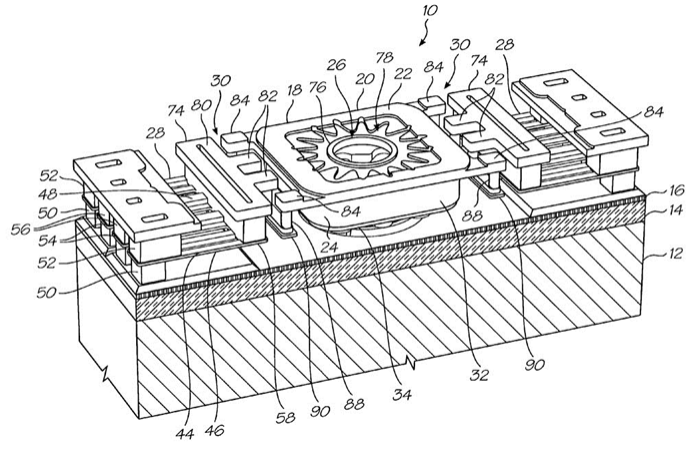

FIG. 2: From Silverbrook patent US 7,524,033.

printing systems.

The developers of this technology have protected it with an extraordinarily large number of patents, some of which describe other forms of actuation. For example, US patent 7,524,033 describes the use of thermally actuated flexures to move the nozzle towards the ink cavity, thus creating enough pressure to eject a drop (Figure 2, [8]).

\section{Electrospray and Tonejet}

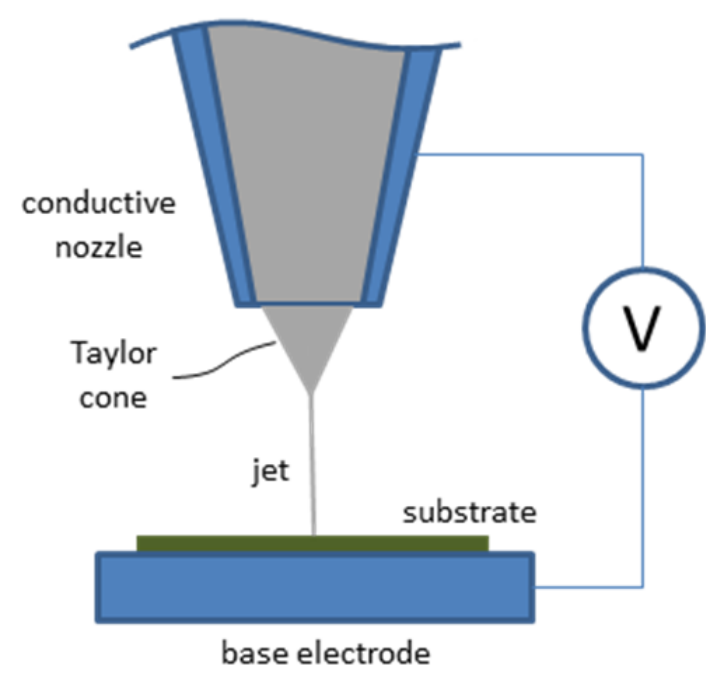

FIG. 3: (Colour online) Taylor cone and jet.

The electrospray technique, also known as electrostatic or electrohydrodynamic (EHD) printing is one of the techniques which can produce small drops from larger nozzles. It has been known for many years that an electric field can draw liquid from a nozzle, [9-11]. Under the right circumstances, the liquid in the nozzle will form a conical shape (a Taylor cone) and a fine jet or stream of drops will be drawn from the tip of the cone, as illustrated in Fig. 3. Other drop-forming modes are possible such as continuous jetting, dripping and, at the highest electrical potential, one or more jets emerging from the nozzle edges, [12]. The major advantage of the electrospray method is its ability to create very fine jets from larger nozzles. A significant challenge is the modulation of the jet to create ink deposits on demand. This is possible by pulsing the voltage, although the need to move the liquid from the normal meniscus position to the Taylor cone shape can limit the frequency at which this can be done, [13].

Park et al. created a system using small nozzles and a moving table to deposit extremely small quantities of ink in a controlled way, [14]. Nozzles of internal diameter $30 \mu \mathrm{m}$ were able to produce drops on the substrate with diameters of $\sim 8 \mu \mathrm{m}$ while tests with nozzles as small as $300 \mathrm{~nm}$ produced spots $\approx 240 \mathrm{~nm}$ in diameter.

A variation on the electrospray technique is the Tonejet system, $[15,16]$. In this case there are no nozzles but instead a comb-like array of pointed electrodes (ejectors) beneath the surface of a liquid containing charged toner particles suspended in an inert carrier such as isoparaffins. An electric field between any of the ejectors and an external common electrode causes the charged toner particles to be concentrated and ejected in a liquid drop. The concentration of toner in the ejected stream is much higher than that in the bulk liquid. The amount of ink ejected can be controlled by the time for which the field is applied so that there is a grey-level capability for each pixel.

\section{Acoustic drop generation}

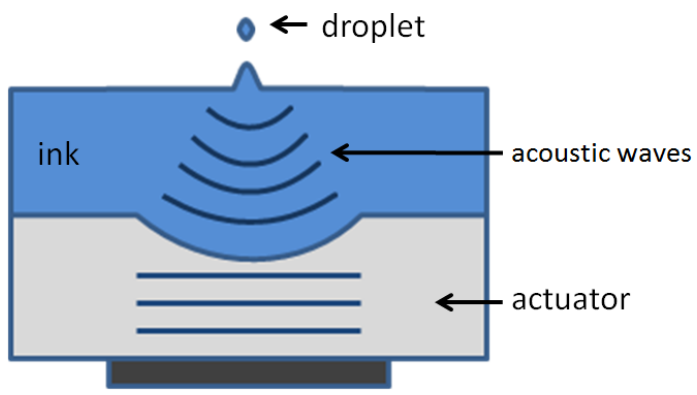

FIG. 4: (Colour online) Acoustic energy, from a transducer, focused by an acoustic lens on to the surface of a liquid causes a drop to be ejected.

With this technique a burst of acoustic energy is focused to a spot on the surface of a liquid causing a drop to be ejected $[17,18]$. This has the advantage that no nozzle is required to produce a small drop. Various methods of focusing the acoustic energy can be used, including shaped acoustic lenses (Figure 4) and acoustic Fresnel lenses. 


\section{Cavity collapse}

This novel way of generating small drops from large nozzles [19] uses phenomena akin to those which produce Worthington jets [20] and the ejection of material by shaped explosive charges [21]. A negative pressure pulse is applied to ink in a cavity behind a nozzle in which a meniscus is maintained. The negative edge of the pressure wave retracts the meniscus, overturning the surface in such a way that a cavity is created. This cavity is then forced to collapse under the action of the positive edge of the pressure pulse. This violent collapse produces a thin jet that eventually breaks up and produces a drop which is significantly smaller than the nozzle: for example a $110 \mu \mathrm{m}$ drop can be formed from a $2.2 \mathrm{~mm}$ diameter nozzle.

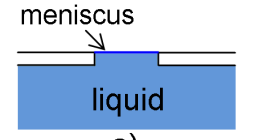

a)

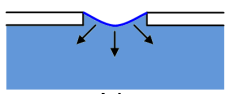

b)

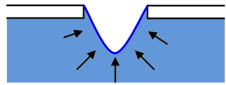

c)

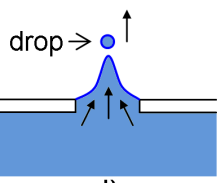

d)

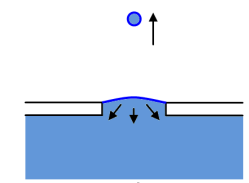

e)

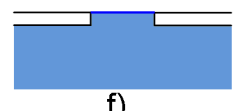

f)
FIG. 5: (Colour online) Principle of jetting by the cavity collapse method: a) steady state, b) meniscus moves into the cavity behind the nozzle, c) the extended meniscus collapses, d) a high speed jet is formed and a droplet produced, e) the meniscus retracts, and f) the system regains its initial condition.

Figure 5 illustrates the process which has been shown to work with a number of different printhead designs. This approach may be particularly useful for printing liquids with very high particle loadings or indeed where the particle sizes are large and could not be printed using a conventional system because the nozzle would become blocked.

\section{PRESENT AND FUTURE APPLICATIONS}

Fig. 6 shows a broad classification of the printing business today. It presents a much-simplified list, and many of the classifications can be broken down into several deeper levels.

The first point to be made about these applications is that they are nearly all aimed at producing a graphical, visible image. The only exceptions are printed circuit board (PCB) manufacturing, the deposition of functional electronic materials, where (typically) screen printing is currently used to create resistors, capacitors and to pattern some active materials such as phosphors, batteries and organic photo-voltaics, and the emerging field of $3 \mathrm{D}$ printing. From the standpoint of the business community, the question posed by Fig. 6 is: what determines the areas which adopt inkjet, and the speed at which it happens?

The motivations for inkjet printing for graphics and text are obvious, and have been widely recognised for the past two decades. However, the adoption of the technology into new application areas seems to be driven by different aspects. The broad trends driving the adoption of digital imaging (not just inkjet) are: $i$ ) targeted advertising and promotional literature, $i i$ ) personal choice of (for instance) decorative products, and iii) reducing scrap and stock levels (the amount of resources held by industry to feed manufacturing processes).

Although inkjet was originally conceived as a digital printing process, the features needed for printing also have the potential to provide the foundations of a generic manufacturing process in which a functional fluid is delivered in very precise quantities at virtually any location within a defined working volume. For graphics printing, the functional fluid is an ink, delivering defined amounts of colour to defined positions on a two-dimensional substrate. However, it has now been well demonstrated that inkjet technologies can also be used to form fully threedimensional objects by printing: a type of additive manufacturing. In the manufacturing context, it is helpful to classify printing processes into three types: $2,2 \frac{1}{2}$ and 3 dimensional printing. These are illustrated in Fig. 7. 2D printing, where the height of the deposited material is irrelevant compared with the width of the printed pattern, is widely used for graphics. In comparison, in $2 \frac{1}{2} \mathrm{D}$ printing height variations are important but only in regions defined by a $2 \mathrm{D}$ printed plan. In contrast, $3 \mathrm{D}$ printing processes can freely create features with accurate control in three orthogonal directions, as shown in Fig. 8. To employ inkjet as a manufacturing process, the definition of an ink must be broadened to include any fluid that can be converted to a solid with appropriate properties, and the operation of the printing machine needs to be extended to at least $2 \frac{1}{2} \mathrm{D}$ to allow for the building up of a solid structure in multiple layers. When this is used to generate models of 3D objects from relatively low performance materials, it is called Rapid Prototyping, an example of which is shown in Fig. 9. This method has now been established for several decades and is routinely used as part of engineering design, modelling and visualization processes. The logical extension to additive manufacturing, implying the use of materials whose properties are appropriate to the function of the final device, has been developing in recent years. In particular, electrical circuits have been built by inkjet printing and compete with conventional printed circuit manufacturing particularly in respect to the number of layers. The printing of conductive layers on to flexible and transparent substrates has recently been demonstrated, a process unattainable by established methods, [22] and in chapter VIII in [1].

Additive manufacturing has also been called "Desk- 


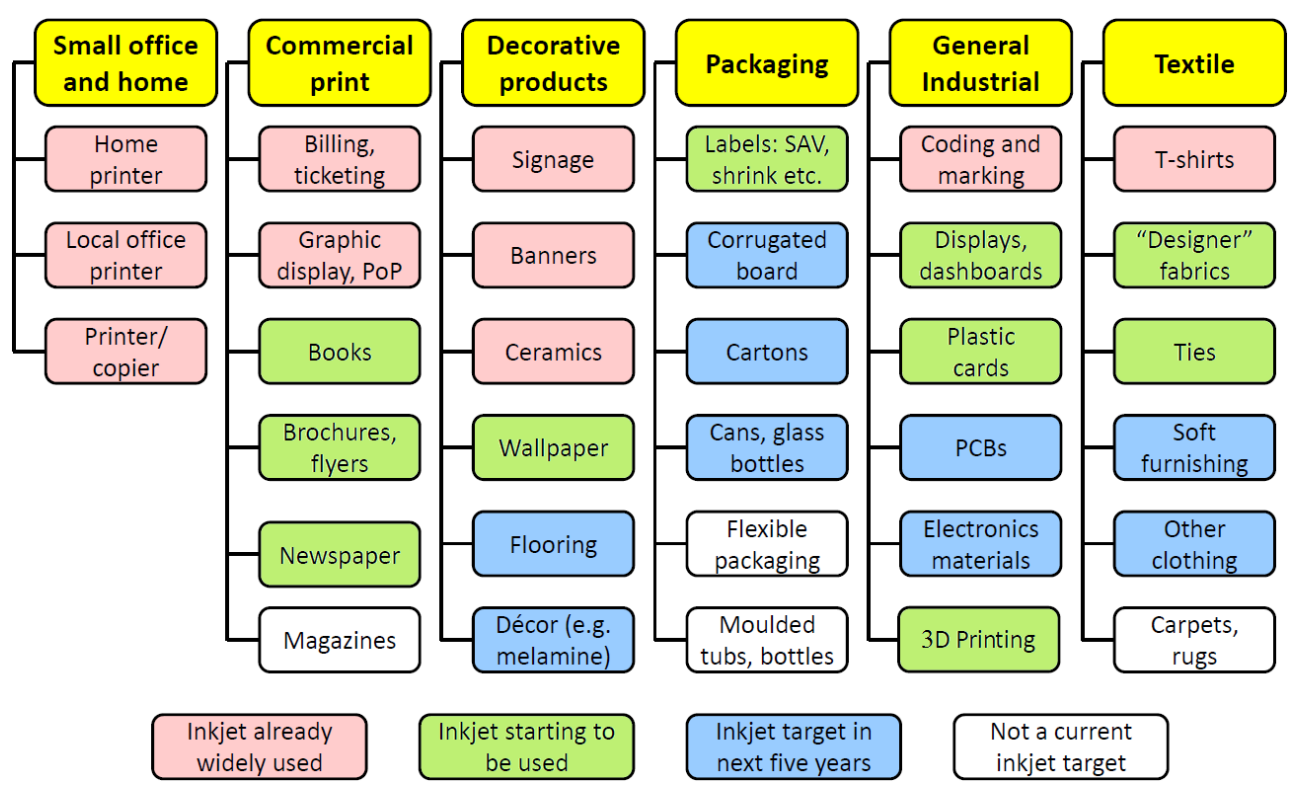

FIG. 6: (Colour online) The print universe.

top Manufacturing" by analogy with desktop printing. The term is probably not really appropriate for inkjetbased methods, because the inks required to form functional materials, and the post-processing that they often require, make it unlikely that they will readily be compatible with small-scale, desktop use. However, the attractions of additive manufacturing are similar to those of digital printing: no mould is required and no limits are placed upon pattern repeat or complexity, short runs are economical, inks can be changed rapidly, the unit of manufacturing can be small with relatively low capital investment, and, most importantly, the process is additive rather than subtractive, implying much less material waste and a more sustainable process. There are, therefore, strong commercial incentives to develop additive manufacturing.

Generically, the additive manufacturing process consists of producing multiple planar layers composed of at least one, and commonly at least two, different materials. One material is a precursor of the final functional material, whilst another may be a disposable filler or support material which maintains the planarity of each layer in regions where there will be empty space in the final object.

Once the appropriate number of layers has been printed, a subsequent process converts the wanted material into its final form, and the (unwanted) filler is removed, often to be re-cycled in the same machine. One process (typically that historically used for rapid prototyping) uses a powder both as the filler and the final material. This is laid down as a homogeneous layer, and inkjet printing is used to deliver a fluid which acts as a binder, as a sintering promoter or inhibitor. Either during or after the printing process, the powder is sintered; the unsintered material is removed to expose the final product. Alternatively, inkjet printing can be used to print both the final material and a separate filler. Often some form of post-processing is required to consolidate and convert the material into its functional form as the final object.

Perhaps the most ambitious form of additive manufacturing has been devised in the context of regenerative medicine, in which biological materials, including stem cells, are used to grow replacement organs. In this case, the filler material may be a wax or a porous material, the active material includes the stem cells, and the postprocessing consists of growing the cells to form the organ. Other biological applications include the direct printing of tissue to reproduce bone structures, but these technologies are still limited to a very narrow set of materials, [23].

Another non-conventional application of inkjet is in spray cooling where droplet evaporation is used to dissipate heat from electronic chips and processors. Conventional spray cooling nozzles are not suited to modern microprocessors due to its large size nozzles and uniform printing patterns, [24] and [25]. In contrast, inkjet technologies present a solution by delivering liquid droplets to hot-spot regions under digital control. This process promises heat dissipation rates at least ten times superior to conventional cooling techniques, [26] and [27].

The questions addressed in the next section are: why has the penetration of inkjet into graphical printing applications been so slow and inconsistent, and what barriers exist to its wider exploitation in additive manufacturing? 


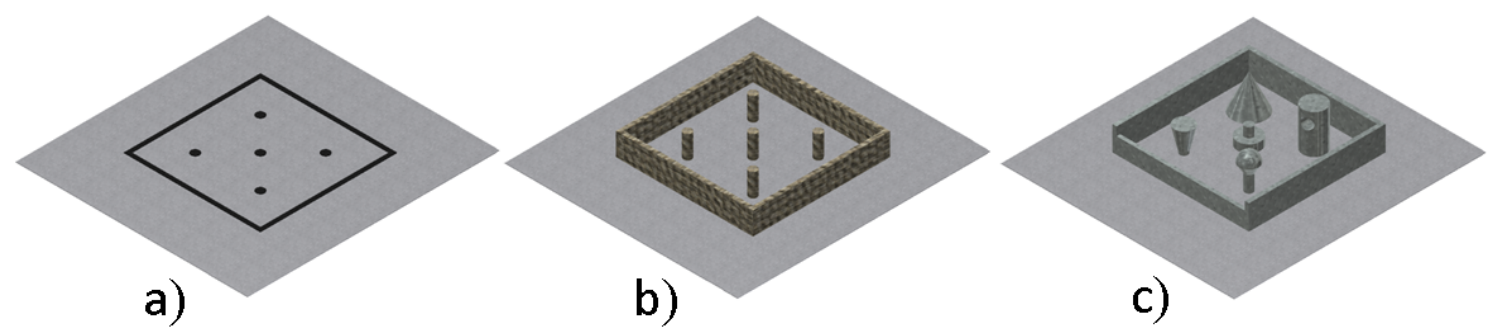

FIG. 7: (Colour online) The different printing scenarios: a) conventional 2D, b) $2 \frac{1}{2}$ application, and c) 3D printing.

\section{BARRIERS TO THE FASTER ADOPTION OF INKJET FOR PRINTING}

Even in the printing of graphics, inkjet has fierce competition from long-established technologies such as screen printing, flexographic printing and lithographic methods. The current capabilities of industrial inkjet processes need to be enhanced to meet the requirements already satisfied by competitive technologies. Some of the constraints are: ink performance, substrate handling problems, printing speed, reliability and cost. These are discussed below.

\section{A. Ink performance}

Conventional industrial printing has developed over many years, and is now superbly cost-effective. It has evolved to meet increasingly demanding and specialised requirements that typically include consumer health and safety, process handling requirements, shelf life, odour and taint as well as image quality. As a result, specific print applications have developed not only their own sets of inks, but also entire process and equipment sets to match their particular requirements. The industry supply chain becomes suited to a specific application, and it is hard for an entirely new process like inkjet to break into this market. Flexible packaging provides a good example of an industry that would greatly benefit from an economic solution for print runs of 0 to $5 \mathrm{~km}$, but for which there is currently no viable inkjet (or indeed other digital) solution. The equipment used is typically central impression flexography (commonly called CI flexo) where the print units are arranged around a large drum to maintain registration between colours in a stretchable material like flexible packaging film. CI flexo provides high-speed, low-cost printing, but carries some disadvantages such as long loading times (i.e. it takes half an hour to change images) and high costs (e.g. a set of printing plates costs $\sim$ US $\$ 1,000$ ).

The main barrier to implementing inkjet methods is the requirement for an ink that is safe to ingest, has no odour, low migration of monomers and other components, satisfactory abrasion resistance for the packaging and distribution process, ability to heat seal, pierce and die-cut without chipping, while still providing the intense colours and high definition needed for primary retail packaging on a supermarket shelf. It has taken decades to develop such inks for flexographic printing, but achieving these properties is much harder within the constraints of inkjet ink formulations.

For inkjet printing, substrate speed is still somewhat restricted, although this is partly determined by the fact that the volume of ink required to achieve the required colour density is much greater than for traditional presses. If inkjet processes could lay down more pigment and less carrier fluid (and spread it sufficiently thinly) then they would challenge all conventional printing methods. A potential application would be to provide distributed printing, for example on site at the local book store or even at the newsagent, thus obviating the need for costly storage and transport of large stocks of printed material, with the benefit of making all titles in principle available at any point of sale.

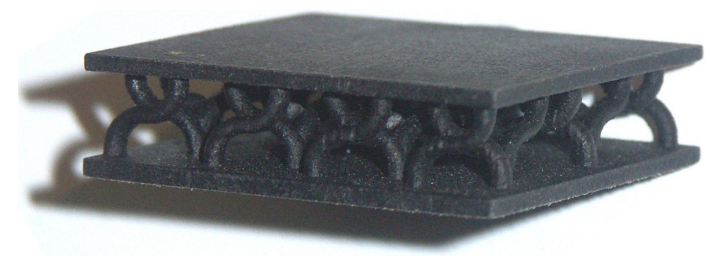

FIG. 8: (Colour online) Honeycomb structure made from polyamide using a powder bed and an inkjet-assisted sintering method.

In drop on demand systems, ink rests in the nozzle between ejection events, for times which depend on the image that is being printed. Ejecting a droplet can be problematic if the ink dries in the nozzle and the force of the next droplet ejection event is not strong enough to remove the dried deposit. Drop on demand systems therefore often use inks that are relatively slow-drying when compared to CIJ. However as drop on demand droplets are much smaller than CIJ, the drying times on the substrate are still reasonable.

The maximum viscosity of an ink which can be used in inkjet printing restricts the amount of solids which it can contain, when compared with competing technologies such as screen or offset printing which can handle more viscous inks. Inkjet inks therefore lack some of the opacity and brilliance displayed by competing technologies. 
For printing, it is the pigment which governs the final functionality: the higher the pigment content, the better (in principle). Typically, inkjet inks contain $<10 \%$ pigment, whereas offset lithography inks contain $>25 \%$ pigment. If inkjet printing could deliver higher solids loadings, then the coverage achieved would be greater and the throughput would be proportionally increased. To cover the paper fully with a $10 \%$ pigment loading requires about $6 \mu \mathrm{m}$ of ink thickness, compared to offset lithography which uses only $2 \mu \mathrm{m}$ thickness. Consequently, there would be a gain of around a factor of 3 in productivity if inkjet were able to use the higher pigment loading employed in offset. This would make the two processes comparable. However, at the same time as increasing the loading, the drop must spread further on landing, delivering the thinner film. This is not what happens naturally and, consequently, represents a further hurdle for the process to overcome. Developing appropri-

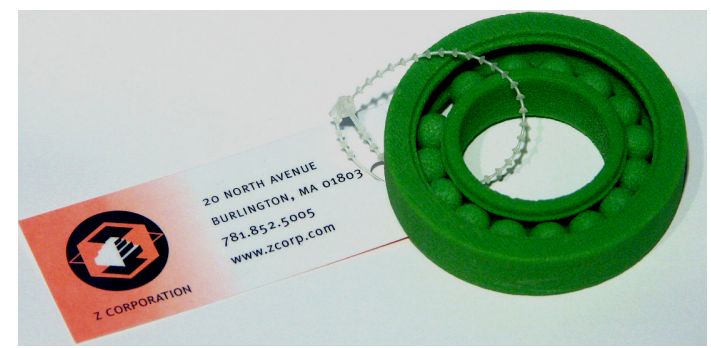

FIG. 9: (Colour online) Example of a rapid prototyping process; a bearing printed by the Z-Corp process using nonfunctional materials.

ate inks is the biggest challenge facing inkjet, particularly in order to extend its range of applications. The delivery mechanism places constraints upon the properties of the fluid, and only in very recent times has the understanding of the rheology and the way in which it affects the formation of droplets developed to the point where competent predictions can be made about which inks will perform well, [3]. Traditionally, empirical methods have been used in both ink development and ink production. The inkjet world is littered with horror stories of how some apparently trivial change to an ink formulation has caused failures ranging from simple loss of quality through to catastrophic clogging of printheads. Inkjet nozzles operate in regimes that are not capable of measurement by conventional methods. In particular, the shear rate in a nozzle is of the order of $10^{6} \mathrm{~s}^{-1}$ which is many orders of magnitude greater than that attainable in conventional rheometers. In addition, the time taken for the drops to break off from the nozzle is comparable with the relaxation times of long chain polymers so that elastic effects can produce dramatic changes to the way the drops form, if indeed they form at all. Polymers are commonly incorporated into ink formulations to stabilize particle dispersions and to control the behaviour of the drops once they have landed. In some instances, the polymers themselves are the functional part of the fluid, for example in UV-curable inks or in fluids designed for direct deposition of the final material in additive manufacturing. The rate of expansion of surface area as the drop is formed challenges the rate at which surface active molecules can diffuse to the liquid surface, making the behaviour of surfactant-containing inks difficult to predict. Currently, the functional requirements of inks are dominated by the jetting process. However, in future, the behaviour of the drop after landing will become increasingly important. In printing, the dot shape is important for the perceived quality of the product, and the increasing range of substrate materials will place demands on the landing and spreading behaviour.

\section{B. Substrate handling}

Conventional printing methods often use a roller pressing on to the substrate to transfer the ink. In some cases, it is an advantage to use a non-contact print method such as inkjet: delicate substrates used in electronics and displays can be contaminated by contact with an inktransfer roller. More often though, it makes the physical handling of the substrate more difficult. A typical example is corrugated board, increasingly used for "shelf ready" packaging on supermarket shelves and for other retail environments where high quality print is needed. The most commonly used printing method is aqueous flexography, in which the board is taken from a stack through the entire print/dry/stack process using roller transport. Some problems exist due to nip-roller contact, but registration between colours can be accurately maintained. When using inkjet, nip-rollers can be used up to the inkjet print station, but after that the transport must rely on contact only with the unprinted side. In addition to this complication, corrugated board warps markedly in response to variations in humidity, and often sustains damage to its edges when the stack is strapped up and transported. It is very difficult to maintain jetting distances of $\approx 1 \mathrm{~mm}$, which are required for good quality inkjet print, when handling such materials. Frequent substrate strikes occur. Problems with corrugated board and other fibrous substrates also occur because fibres are shed in handling, and cause nozzle reliability problems.

\section{Printing Speed}

Inkjet has a number of advantages over conventional processes, mostly arising from a combination of its versatility and the digital control. These lead to production rate advantages such as reduced turnround times, reduced inventory of patterns and the ability to print long pattern repeats. However, its rate of delivery of ink is generally lower than that of competing conventional processes, and there is continual pressure to increase its throughput. Inkjet is in fact replacing most screen printing equipment in the screen graphics market where it 
achieves comparable speeds. However, in other areas of printing, inkjet lags significantly on speed: the typical linear throughput of inkjet is of the order of $1 \mathrm{~m} / \mathrm{s}$, compared with offset lithography at $5 \mathrm{~m} / \mathrm{s}$ or flexography at $10 \mathrm{~m} / \mathrm{s}$. Industrial screen applications have not been affected by inkjet in applications such as the manufacturing of PCBs for car dashboards, and pots for food. In neither of these market areas has inkjet made significant inroads, despite its other advantages.

The quality achievable by inkjet printing is clearly good enough for some graphical purposes: one only has to look at the complete displacement of chemical-based photography by inkjet. However, this quality is currently achieved at the cost of speed, and to address this, inkjet needs to produce smaller drop sizes whilst maintaining the volume flow rate. In most other applications, inkjet is not yet comparable to most flexo, lithographic and gravure technologies. This presents extensive challenges both for the printhead maker and for the ink developer.

\section{Reliability}

All forms of inkjet printing technologies appear to be susceptible to the formation of small drops in addition to the main printing drops. These drops are termed satellite droplets, and their formation undermines the reliability of inkjet printing systems.

Drop on demand systems tend to form satellite droplets more readily than continuous inkjet. In contrast to the continuous inkjet systems therefore, avoiding satellites is more problematic and is often achieved by using an optimised drive waveform or an ink formulation with non-Newtonian properties.

In drop on demand technologies the satellite droplets are very small and do not have enough momentum to travel towards the substrate. They are often attracted to the nearest surface, such as the inkjet nozzle, and if they contaminate the nozzle then the formation of the main drops is affected resulting in printing failure.

In continuous inkjet systems satellite droplets have a higher charge to mass ratio than the main drops due to the charging geometry. This higher charge to mass ratio often results in the droplets being attracted to the charging plates within the heads, leading to the head becoming contaminated with ink and ultimately to the failure of the head when the build-up of ink interferes with the ink being delivered on to the substrate.

Modelling of fluid behaviour in continuous inkjet systems with Newtonian inks shows that both satellite-free and satellite-forming modes of operation are possible, as shown in Fig 10. Satellites can be avoided by choosing the right combination of jet velocity, frequency, viscosity and drive amplitude. Continuous inkjet systems therefore need careful control of operating parameters and ink composition in order to behave reliably.

Reliability is also affected by the ability of the printer to start and stop. In continuous inkjet printers this tends
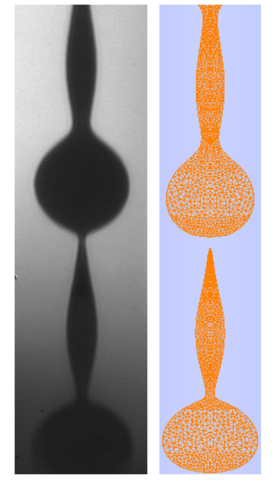

a)

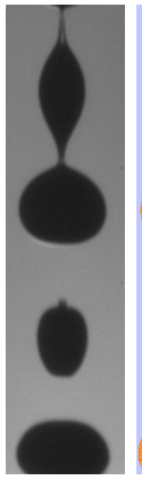

b)

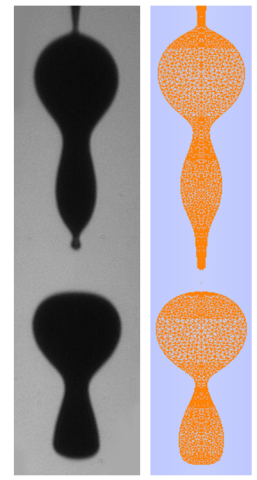

c)
FIG. 10: (Colour online) Comparison of jet breakup shapes in three continuous inkjet processes. Experiments (left) were recorded by strobe-shadowgraphy of jets produced by a commercially availably Domino plc printer. Simulations (right) were performed using the Lagrangian method presented in [28]. Conditions as follows: a) modulation amplitude of $40 \mathrm{~V}$ and jet velocity of $0.6 \mathrm{~m} / \mathrm{s}$, in b) modulation of $140 \mathrm{~V}$ and jet velocity of $1.7 \mathrm{~m} / \mathrm{s}$ and in c) modulation of $180 \mathrm{~V}$ and jet speed of $2.1 \mathrm{~m} / \mathrm{s}$. The only satellite-free case is c), where the filament recombines into a single drop.

to be an occasional event when the device is first switched on. As the jet velocities are so high, any amount of dried ink present in the nozzle tends to cause the printer to start very unreliably. Elaborate routines have therefore been devised to flush and clean the nozzle so that the printer will start reliably, and, by leaving no ink in the drop generator when the printer is switched off, also stop reliably. Continuous inkjet printers can therefore be designed to print inks with very volatile solvents giving very short drying times, even though the drops are quite large.

\section{E. Quality/cost trade-off}

Current high volume print applications such as glossy magazines and primary packaging attain superb print quality levels at very low cost. Offset lithography and gravure have been developed and used over decades, and flexography now approaches them closely in terms of quality and reliability. These technologies and inkjet compete in a market where a single characteristic may be key to determine which method is best suited for a particular application. Comparisons in terms of speed and costs are shown in Tables 11 and 12 .

Compared with inkjet, changing the image in these methods is slow and expensive, but the three processes differ greatly:

- A set of offset lithographic plates might cost $\sim$ US $\$$ 100 and take half an hour to set up.

- A set of flexographic plates might cost $\sim$ US $\$ 1,000$ and take 20 minutes to set up.

- A set of gravure rollers might cost $>$ US $\$ 10,000$ and take an hour to set up. 


\begin{tabular}{|c|c|c|c|c|}
\hline Technology & Print speed & Print quality & Changeover time & Typical set-up scrap \\
\hline DoD inkjet & $1-3 \mathrm{~m} / \mathrm{s}$ & $\mathrm{V} \sqrt{ } \sqrt{ }$ & Zero & Zero \\
\hline CIJ inkjet & $5 \mathrm{~m} / \mathrm{s}$ & $\mathrm{VV}$ & Zero & Zero \\
\hline Lithography & $5 \mathrm{~m} / \mathrm{s}$ & $\mathrm{V} \sqrt{ } \sqrt{ } \sqrt{ }$ & 20 minutes & 200 sheets \\
\hline Flexography & $5 \mathrm{~m} / \mathrm{s}$ & $\mathrm{V} \sqrt{ } \sqrt{ }$ & 40 minutes & 500 sheets \\
\hline Rotogravure & $8 \mathrm{~m} / \mathrm{s}$ & $\sqrt{ } \sqrt{ } \sqrt{ } \sqrt{ }$ & 60 minutes & 1000 sheets \\
\hline
\end{tabular}

FIG. 11: (Colour online) Comparison of printing technologies in terms of performance.

Because flexography plates are often re-used, the plate cost is not significant as may seem from the figures above. The trend to smaller print runs has therefore favoured flexography. Sheet-fed offset, which is quicker to set up than web-fed offset, has not been affected as much as gravure. In response to the threat from digital printing, all equipment suppliers have worked hard to reduce the time, labour and amount of substrate scrap involved at set-up.

Inkjet can offer a cost-competitive alternative to these print processes, but is still not able to deliver the print quality that customers expect. There are three main causes that impair inkjet print quality:

a) Print direction "banding". Any systematic irregularity in inkjet printing will result in defects across the print direction. Typical sources are variability in nozzle straightness, drop size or drop velocity; misregistration due to weaving of the substrate; and missing or deviated nozzle jets.

b) As noted above, inkjet inks have constraints on their formulation which are not present for conventional inks. Their low viscosity limits the size, quantity and composition of the pigments that can be held in suspension, and also limits the types of carrier materials that can be used (e.g. UV curing monomers rather than the oligomers typical of flexography). Pigments are held in suspension, and prevented from sticking together by polar coatings so that they repel each other, but larger particles (with a smaller ratio of surface area to volume) tend to agglomerate in a low viscosity fluid. In contact printing (flexography and lithography) this is not a problem because the particles are not mobile, but inkjet inks flocculate easily. On the other hand, if pigment particles are ground too finely, they tend to increase the viscosity. In addition, dense pigments also pose problems. White inkjet ink contains about $20 \%$ titania $\left(\mathrm{TiO}_{2}\right)$ with a very small particle size, while screen inks contain about $60 \%$ of larger particles, and hence have greater opacity. Higher contents of titania in inkjet are impossible to stabilize because the dense particles tends to settle out of suspension quickly in a low-viscosity ink.

Jetting stability enforces tight control of composition and rheology on inkjet inks, whereas inks for conventional printing have a wider operating window.

c) Satellite drops cause problems on the printed product itself, but also because being separate from the main ink stream they can end up on nozzle plates or on other parts of the printhead so that nozzle reliability is affected, as noted above. These small satellites are easily dispersed by aerodynamic effects or static charges, are transported by stray air currents induced by the substrate movement and are eventually deposited in undesirable locations. This problem is particularly acute in electronics and display applications, where bridging between tracks and poisoning of adjacent cells can occur, [29].

\section{CHALLENGES FOR INKJET-BASED ADDITIVE MANUFACTURING}

In this section, the technical hurdles that stand in the way of further development of inkjet-based additive manufacturing processes are examined. In practice, some of these obstacles apply to printing itself as well as to additive manufacturing, but the latter stretches just about every aspect of the underlying technology: i) finding the right ink, ii) delivering it sufficiently rapidly, and iii) controlling the final form. By overcoming some of these aspects, inkjet could emerge as an alternative technique for manufacturing and could also be used to make shapes that cannot be made by traditional methods such as moulding or machining.

\section{A. Limits to printability}

For additive manufacturing, perhaps more than for most other applications of inkjet printing, the limitations on the fluids which can be printed are critical. The fundamental physical limits to printability imposed by the properties of the liquid have been discussed by [30] and [31]. The vertical axis represents the Ohnesorge number, Oh, a dimensionless group determined by the viscosity, surface tension and density of the fluid, as well as the diameter of the jet or drop. For high values of $\mathrm{Oh}(\mathrm{Oh}$ $>\sim 1$ ), viscous forces prevent the separation of a drop, while for low values $(\mathrm{Oh}<\sim 0.1)$ the jet forms a large number of satellite drops.

The jet must also contain enough kinetic energy to be satisfactorily ejected from the nozzle, and this condition 


\begin{tabular}{lllll}
\hline Technology & Typical ink cost & Typical plate cost & Advantages & Disadvantages \\
\hline DoD inkjet & $40 \mathrm{USD} / \mathrm{kg}$ & Zero & Variable data & Slow \\
CIJ inkjet & $25 \mathrm{USD} / \mathrm{kg}$ & Zero & Variable data & Limited ink types \\
Lithography & $10 \mathrm{USD} / \mathrm{kg}$ & $100 \mathrm{USD}$ & Mature technology & $\begin{array}{l}\text { Most suitable for } \\
\text { coated paper }\end{array}$ \\
Flexography & $5 \mathrm{USD} / \mathrm{kg}$ & $1,000 \mathrm{USD}$ & $\begin{array}{l}\text { Able to use many } \\
\text { types of substrate }\end{array}$ & High ink waste \\
Rotogravure & $5 \mathrm{USD} / \mathrm{kg}$ & 10,000 USD & Long runs & Expensive set-up \\
\hline
\end{tabular}

FIG. 12: (Colour online) Comparison of printing technologies in terms of cost and versatility.

is represented by the lower diagonal line. The horizontal axis of the diagram shows the Reynolds number, Re, a dimensionless group determined by the velocity, drop size, density and viscosity of the fluid. If the drop speed is too high then it may splash on impact, leading to poor control of the final position of the material, and hence to poor resolution; this will occur for conditions above the upper (broken) diagonal line. The limits of printability therefore lie within the unshaded area in the diagram. These limits apply to simple viscous fluids, but many liquids of practical interest for additive manufacturing, either as binders or as materials for direct deposition, are non-Newtonian which imposes further constraints. For example, the presence of even very small concentrations of long-chain polymers in solution can prevent satisfactory printing, while much larger concentrations of polymers with lower molecular weight can be tolerated, [32]. A further constraint on the fluid is present in thermal drop-on-demand printing, where the pressure pulse which ejects the jet is generated by the vaporization of a small volume of the liquid, which must therefore have a low boiling point. Thermal DoD printing therefore has very limited applicability to additive manufacturing.

\section{B. Resolution and surface definition}

For both printing and additive manufacturing, one aspect of quality is determined by the resolution, in effect the number of dots $/ \mathrm{m}^{2}$. This has long been considered a critical factor in graphical printing, but is, in fact, not the most important issue. The eye is limited as to its resolution, and once that limit has been achieved, the brain does a remarkably good job of interpolating both to perceive uniform grey levels by averaging sparse dots, and also to create the illusion of smooth edges. These impressions can be further improved by inserting small dots between larger ones to simulate the weight and position of the line. Again, the brain performs the appropriate interpolation and perceives the result as a smooth, hard edge. In practice, another aspect of quality is determined by the accuracy with which the droplets are placed on the substrate, primarily the accuracy relative to others of the same "colour", less so to those of different "colours".
However, in additive manufacturing, it is necessary to bond all the small volumes of material together to form a continuous 3D body with smooth surfaces. This requires that oversize dots are available as well as undersized, the oversize ones being used to ensure that the smaller ones are appropriately connected. Visual perception is of no help in this situation and, instead, the fluid properties themselves must be exploited to generate the smooth and continuous surfaces. This requires a much greater level of control than for graphics printing, and in turn imposes a greater requirement for understanding the process, for manipulating the material properties and for controlling the placement of the drops.

The most obvious example of this is the printing of conductive tracks for electronics. Separated dots are of no functional use in this application, and in many instances the actual functionality of the tracks is defined as much by the edges as by the bulk. Fully continuous, smooth-edged tracks at virtually arbitrary spacings can be printed using multiple drop sizes, including oversized ones, and involves a completely new set of considerations in comparison to graphics printing.

Volatile inks are often used in additive manufacturing processes. The volatile carrier evaporates to leave the functional material distributed in some manner over the landing region of the drop. Non-linear processes, such as the Marangoni effect, can give rise to an extremely uneven distribution of the final material and this is a key issue for some types of manufacture. One example is the manufacture of polymer LED display screens. Inkjet is currently being used in such processes because of its great accuracy and repeatability of delivery, and, in principle, could replace almost all of the manufacturing steps for screen production. However, the uneven distribution of the material after drying poses problems for further developments.

\section{Printing Speed}

For additive manufacturing, speed is a critical issue because the three-dimensional structures are built up in multiple thin layers. To compete with conventional processes, each layer must be printed as quickly as possi- 
ble. Inkjet throughput can be increased by a number of methods: increasing the number of nozzles, increasing the drop frequency, and increasing the pigment or functional material loading of the ink.

\section{Number of Nozzles}

Whilst notionally straightforward, increasing the number of nozzles is not in practice very easy. The larger the number of nozzles, the more challenging become the reliability issues. Adding more rows of nozzles also creates quality issues such as placing higher demands upon the accuracy of positioning of individual drops and on the stability of the substrate. Machine costs increase rapidly with increasing nozzle count. However, the large numbers of nozzles now being incorporated into larger printing machines offer total delivery capacity which is of the right magnitude for large-scale additive manufacture. A typical 1,000 nozzle inkjet printhead is capable of delivering approximately $1 \mathrm{t}$ of ink per annum. Printers now use anything up to 36 such heads, and could therefore compete, in principle, with medium-sized injection moulders. In practice, though, the low solids content of the inks would reduce this rate substantially.

\section{Drop frequency}

Drop frequency is limited by the physics of the process. Smaller drops can be produced more frequently, but with no net improvement in volume printing rate (although this is key to quality in terms of resolution of the print). The central issue here is the replenishment rate (the rate at which the nozzle refills once a drop has been delivered). Drop on demand inkjet exploits non-linear effects in the nozzle to create a pumping action. As the meniscus moves backwards and forwards, so the nozzle impedance changes and suitably chosen pressure waves generate a net forward motion (surface tension also helps with replenishment, but is relatively very slow). There seems to be a limit of delivery rate of around $1 \mu \mathrm{l} / \mathrm{s}$ for each nozzle, though this is not achieved in practice.

Continuous inkjet (which uses external pumps) can achieve higher volume delivery rates, but with lower resolution and with greater constraints on the type of ink which can be used.

\section{Increased Ink Solids Content}

For additive manufacturing, the issue of pigment loading can be even more critical than for graphical printing. In most cases, the carrier fluid contributes no functionality to the final product, and indeed has to be removed, leaving the solids content to represent the only valuable part. Consequently, the higher the solids loading, the better. The sintered powder type of additive manufacturing process reduces this problem insofar as the functional material is the powder itself, which is not jetted. However, such powders also have a high void content, and so the density of the final material (without sintering) is limited. Hybrid systems in which the sintering promoter contains precursors of the powder material can achieve both higher final densities and lower sintering temperatures.

\section{Throw Distance}

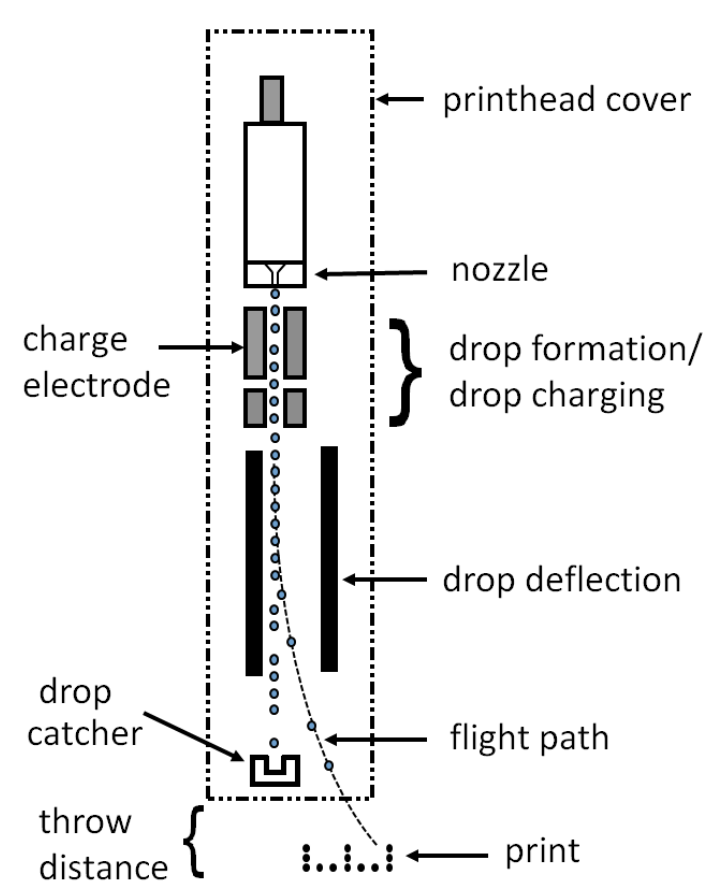

FIG. 13: Throw distance is defined as the distance between the exit of the printhead (generally a slot in a printhead cover) and the substrate. For CIJ a drop usually travels $\sim 100 \mathrm{~mm}$ before landing on the substrate. In CIJ terms, the flight path is the passage of the drop between the nozzle and the printhead exit.

The throw distance is defined as the distance from the printhead to the substrate, as seen in Fig. 13. It is a function of many factors including: the jet velocity, the printhead flight path, the variation in jet velocities across the array, nozzle straightness, drive position errors, air turbulence, printhead perpendicularity and alignment, timing errors, and nozzle pitch variation.

In drop on demand systems, the ink is either ejected from the nozzle by strain in a piezoelectric ceramic, or by expansion of a vapour bubble produced by rapid heating of the ink. Neither method of producing drops gives jet velocities that are as high as those from a continuous inkjet system, [33]. Although drop on demand printers have no intrinsic need for a flight path, as no droplet selection is required, droplet velocities tend to be lower 
than in CIJ, of the order of $6 \mathrm{~m} / \mathrm{s}-12 \mathrm{~m} / \mathrm{s}$. Drop on demand systems also tend to be targeted at resolutions above 300 dpi and therefore produce small drops with little momentum, which also restricts the throw distance. Array printing is also limited by the variation of droplet velocities across the array and substrate uniformity. In order to print acceptably straight lines the droplets usually need to arrive at the substrate with a lateral placement precision better than one quarter of the drop diameter. Lateral deflection in drop on demand system is often ascribed to aerodynamical and electrostatic effects. If the lateral drop velocity is a significant proportion of the average jet velocity then the throw distance needs to be shortened to keep the lines straight. Typically drop on demand heads have throw distances of less than $5 \mathrm{~mm}$, and often as low as $1 \mathrm{~mm}$.

In single jet continuous inkjet systems, the jet velocity is generated by a pressurized ink system forcing ink through a nozzle, resulting in jet speeds of the order of $20 \mathrm{~m} / \mathrm{s}$. The limitation to the throw distance is caused by the need to charge and deflect droplets, see Fig. 13. As the droplets take a parabolic trajectory, another limitation is that the maximum print height is a function of throw distance. Typically continuous inkjet printers can comfortably jet to a throw distance of $20 \mathrm{~mm}$ before the droplets lose their trajectories and give unacceptably poor positional accuracy.

\section{CONCLUSIONS}

In this review, some of the limitations preventing the fast adoption of inkjet in printing fields still dominated by competitive technologies have been presented. Although inkjet is widely used in some graphic applications, it still lacks the quality or speed of more traditional printing methods such as flexography or lithography. The selection of the right technology for a printing application depends on a trade-off between the limitations of the various inkjet technologies. If a high quality printed product is required then a drop on demand system must be used, but there is a cost for this quality in terms of throughput and ink end-user properties. For higher substrate speeds or for irregularly shaped substrates in harsh environments, continuous inkjet technologies may be more suitable. In any case, it is expected that these and other limitations will be overcome by novel new inkjet technologies.

Importantly, inkjet offers other advantages that make it ideal in applications of non-traditional manufacturing where, if achievable, high resolution and high printing speed would open up the possibilities for competitive production of quality objects by the extension of existing inkjet-based additive manufacturing processes. In this context, the potential for additive manufacture is to be more economical and more environmentally friendly than current methods. Manufacture by inkjet presents challenges to chemists and materials scientists to find ways to deliver both structural and functional materials in liquid form and subject to the constraints of the process. In this review, a list of these challenges has also been discussed with the aim of reaching the academic community and communicating some of the specific needs of industry.

Inkjet has been classified by many as an emerging manufacturing technique. Whilst the term desktop manufacturing is probably inappropriate, nevertheless, the prospect for distributed manufacture by inkjet-based 3D printing at local centres is similar to that of the distributed printing model for graphical products, with potentially far more profound effects both socially and economically.

\section{Acknowledgments}

This work was supported by the Engineering and Physical Sciences Research Council (Grant No. $\mathrm{EP} / \mathrm{H} 018913 / 1$, Innovation in industrial inkjet technology). IMH and GDM wish to acknowledge support from the Diginova EU FP7 project.
[1] Hutchings, I.M and Martin, G.D., (2012) Inkjet technology for digital fabrication, Wiley.

[2] Le, H.P., (1998) Progress and Trends in Ink-jet Printing Technolgoy Journal of Imaging Science and Technology, 42, pp. 42-49.

[3] Basaran, O.A., Gao, H. and Bhat, P.P, (2013) Nonstandard Inkjets, Annu. Rev. Fluid Mech., 45, pp. 85-113.

[4] Furlani, E.P., Price, B.G., Hawkins, G. and Lopez, A.G., (2006) Thermally Induced Marangoni Instability of Liquid Microjets with Application to Continuous Inkjet Printing, Proc of NSTI-Nanotech, pp. 534-537.

[5] Gao, Z., Ng, K., Furlani, E., Chwalek, J. and Hawkins, G., (2010) MEMS-Based Microfluidic Devices Proc. of ASME 2010 3rd Joint US-European Fluids Engineering Summer Meeting and 8th International 8th International Conference on Nanochannels, Microchannels, and
Minichannels, pp. 1-6.

[6] Chwalek, J.M., et al., (2002) A New Method for Deflecting Liquid Microjets, Physics of Fluids, 14(6), pp. L37L40.

[7] Silverbrook, K., (2011) Inkjet Nozzle Assembly with Low Density Suspended Heater Element, US Patent $7,980,673$.

[8] Silverbrook, K., (2009) Nozzle Arrangement with Movable Ink Ejection Structure, US Patent 7,524,033.

[9] Taylor, G.I., (1964) Disintegration of Water Drops in an Electric Field, Proc. of the Royal Society of London Series A. Mathematical and Physical Sciences 280(1382), pp. 383-397.

[10] Zeleny, J., (1917) Instability of Electrified Liquid Surfaces, Physical Review 10(1), pp. 1-7.

[11] Winston, C.R., (1962) Method and Apparatus for Trans- 
ferring Ink, US Patent 3,060429.

[12] Cloupeau, M. and Prunet-Foch, B., (1994) Electrohydrodynamic Spraying Functioning Modes: a Critical Review, Journal of Aerosol Science, 25(6), pp. 1021-1036.

[13] Kim, J., Oh, H. and Kim, S., (2008) Electrohydrodynamic Drop-on-demand Patterning in Pulsed Cone-jet Mode at Various Frequencies, Journal of Aerosol Science, 39(9), pp. 819-825.

[14] Park, J-U., et al., (2007) High-resolution Electrohydrodynamic Jet Printing, Nature materials, 6(10), pp. 782789 .

[15] Newcombe, G., (2008) Tonejet: Delivering Digital Printing to the Mass Market, Proc of NIP24 and Digital Fabrication, pp. 402-404.

[16] Newcombe, G. and Goodchild, M., (1999) Method and Apparatus for Ejection of Particulate Material, US Patent 5,992,756.

[17] Elrod, S., et al., (1989) Nozzleless Droplet Formation with Focused Acoustic Beams, Journal of Applied Physics, 65(9), pp. 3441-3447.

[18] Hadimioglu, B., et al., (1992) Acoustic Ink Printing, Proc. of Ultrasonics Symposium, pp. 929-936.

[19] Castrejon-Pita, A.A., Castrejon-Pita, J.R. and Martin, G.D., (2012) A Novel Method to Produce Small Droplets from Large Nozzles, Review of scientific instruments, 83(11), 115105.

[20] Gekle, S. and Gordillo, J.M., (2010) Generation and Breakup of Worthington Jets After Cavity Collapse. Part 1. Jet Formation, Journal of Fluid Mechanics, 663, pp. 293-330.

[21] Birkhoff, G., MacDougall, D.P., Pugh, E.M. and Taylor, G., (1948) Explosives with Lined Cavities, Journal of Applied Physics, 19(6), pp. 563-582.

[22] Delaney, J.T., Liberski, A.R., Perelaer, J., Schubert, U.S., (2010) A Practical Approach to the Development of Inkjet Printable Functional Ionogels-Bendable, Foldable, Transparent, and Conductive Electrode Materials,
Macromolecular Rapid Commun, 31, pp. 1970-1976.

[23] Derby, B., (2012) Printing and Prototyping of Tissues and Scaffolds, Science, 338(6109), pp. 921-926.

[24] Ortiz, L. and Gonzalez, J.E., (1999) Experiments on Steady-State High Heat Fluxes Using Spray Cooling, Experimental Heat Transfer, 12(3), pp. 215-233.

[25] Sharma, R.K, Bash, C.E., and Patel, C.D., (2005) Inkjet Assisted Micro-scale cooling of Electronics, NSTINanotech, 1, pp. 644-647.

[26] Bash, C.E., Patel, C.D., and Sharma, R.K., (2003) Inkjet Assisted Spray Cooling of Electronics, Proc. IPACK03, pp. 1-9.

[27] Vondram, G., et al. (2012) Thermal Performance of Inkjet-assisted Spray Cooling in a Closed System, IEEE ITHERM Conf, pp. 1127-1131.

[28] Castrejon-Pita, J.R, Morrison, N.F., Harlen, O.G., Martin, G.D. and Hutchings, I.M., (2011) Experiments and Lagrangian Simulations on the Formation of Froplets in Continuous Mode, Phys. Rev. E , 83, 016301.

[29] Perelaer, J., et al. (2010) Printed Electronics: the Challenges Involved in Printing Devices, Interconnects, and Contacts Based on Inorganic Materials Journal of Materials Chemistry, 20, pp. 8446-8453.

[30] Derby, B., (2010) Inkjet Printing of Functional and Structural Materials: Fluid Property Requirements, Feature Stability, and Resolution, Ann. Rev. Materials Research 40, pp. 395-414.

[31] McKinley, G.R. and Renardy, M., (2011) Wolfgang von Ohnesorge, Phys. Fluids, 23, 127101.

[32] Hoath, S.D., Harlen, O. G., and Hutchings, I. M., (2012) Jetting Behaviour of Polymer Solutions in Dropon-demand Inkjet Printing Journal of Rheology, 56(5) pp. 1109-1127.

[33] Basaran, O.A., (2002) Small-Scale Free Surface Flows with Breakup: Drop Formation and Emerging Applications, AIChE Journal, 48, pp. 1842-1848. 